\title{
Asignación de surtido, mercado paralelo y enforcement en la cadena de suministro de artículos de gran consumo: Un análisis empírico
}

Fernando Gimeno-Arias y Miguel Hernández-Espallardo

https://doi.org/10.37610/dyo.v0i72.586
Recibido: 14 de Junio de 2020

Aceptado: 11 de Septiembre de 2020

\section{Resumen}

El propósito del artículo es el análisis de la estrategia dentro de la cadena de suministro de artículos de gran consumo. El diseño del canal de distribución es una situación crítica donde se confronta la estrategia con el comportamiento de los agentes. Se observa que la alternativa del distribuidor en caso de disconformidad con el surtido asignado es el recurso al mercado paralelo. El análisis conduce al estudio del efecto de la disciplina del fabricante sobre el canal para hacer cumplir la estrategia de distribución oficial. Las conclusiones son expuestas a partir de resultados obtenidos en un análisis empírico.

\section{Palabras clave}

Cobertura clientes, asignación surtido, distribución oficial, mercado paralelo, enforcement

\section{Introducción}

El diseño de canales de distribución es un aspecto complejo con múltiples elementos a tener en cuenta. Esto se ha traducido en el uso de múltiples enfoques teóricos para su análisis: microeconómico, comportamental, economía política o costes de transacción (Heide, 1994). Progresivamente se han ido simultaneando elementos transaccionales y relacionales, observándose que un aspecto fundamental es el tipo de mercado. Frazier (1999) concluía que, en la distribución de productos de gran consumo, la incidencia de la relación no es observable o tiene escaso impacto. Estos canales están influenciados por otros aspectos de la estrategia de marketing.

En la fase de diseño del canal, los fabricantes con una cartera amplia de productos que pretenden operar en distintos segmentos de mercado han de decidir a quién se dirigen y, en función de estos clientes objetivo, estructuran su distribución mediante distribuidores oficiales especializados por tipo de cliente que ofrezcan un servicio ajustado a la oferta de valor para cada cliente. Esto se hace mediante una política de cobertura de clientes, destinada a mantener una relación cercana con cada tipo de cliente y proveer asistencia técnica especializada asegurándose que sus productos son distribuidos únicamente por intermediarios competentes y cualificados (Coughlan et al., 2006). La situación derivada para cada intermediario especialista es la dotación de un

\footnotetext{
Fernando Gimeno-Arias * fernando.gimeno@um.es

iD https://orcid.org/0000-0001-6301-0685

Miguel Hernández-Espallardo * migher@um.es

iD https://orcid.org/0000-0003-3513-7990

* Universidad de Murcia.
}

surtido especializado en función del cliente que va a atender y el veto a comercializar en estos canales (diseñados ad hoc por tipo de demanda) a aquellos distribuidores no autorizados.

Si la asignación de intermediarios especialistas es una complejidad en el diseño del canal, la presencia de un mercado paralelo que distorsione su orden lo hace más complicado en términos estratégicos. El mercado paralelo consiste en la distribución de productos al margen del canal compuesto por el fabricante y sus intermediarios oficiales y que tiene su origen en el incumplimiento mediante venta no autorizada (Cespedes et al., 1988). Es una situación que se da con cierta frecuencia en canales de gran consumo (Antia et al., 2004), terreno abonado a ello por los altos niveles de transacciones (Corey et al., 1989).

Con las posibilidades de aprovisionamiento que ofrece un mercado paralelo, la restricción de surtido establecida por el fabricante en el diseño del canal puede ser evadida por los distribuidores adquiriendo productos vetados. Hasta ahora, el desabastecimiento de producto ha sido tratado como un problema técnico, por ejemplo, por problemas de producción (Antia et al., 2006), no como una decisión estratégica del fabricante, cuando decide no servir ciertos productos a través de cierto canal de distribución.

Desde el punto de vista del fabricante, la estrategia del canal de distribución oficial necesita ser vigilada y acompañada de actuaciones que impidan comportamientos desalineados. Han de implantarse un conjunto de medidas disciplinarias con el objeto de velar por su cumplimiento (Antia y Frazier, 2001). Estas acciones que el proveedor adopta para hacer cumplir los acuerdos se denomina enforcement (Bergen et al., 1998; Wathne y Heide, 2000). 
Si desde un punto de vista cualitativo el control del mercado paralelo es un aspecto crítico para la implantación de la estrategia de distribución, los datos a nivel cuantitativo aportan una visión más concreta de la dimensión del problema. Las cifras son de tal envergadura que un grupo de fabricantes han dejado a un lado su rivalidad para aliarse frente al mercado paralelo creando AGMA (Alliance for Gray Market and Counterfeit Abatement), alianza en la que la que están presentes fabricantes del prestigio de CISCO, HP, IBM, MICROSOFT, entre otros. Dicha alianza estima que la incidencia del mercado paralelo supone un impacto en sus negocios de 250.000 millones de dólares, así como la pérdida de 750.000 puestos de trabajo (https://www. agmaglobal.org/).

En cuanto al marco de actuación más reciente del mercado paralelo, la economía circular, especialmente en el ámbito de los productos de gran consumo, ha favorecido una cadena de suministro de circuito cerrado (Cao y Zhang, 2019). Situación que se ha visto contrarrestada por la proliferación de las TIC, que ha impactado en dicha cadena abriéndola a la conexión de distintos agentes en todo el mundo a través de la WEB, como se constata en el uso del mercado paralelo que intermediarios como Amazon, eBay, Alibaba, Kmart o Costco hacen para favorecer sus estrategias de suministro, intermediación o venta (Gudigantala y Bicen, 2019). A diferencia del mercado paralelo offline, la tecnología de la información impulsa un mercado paralelo online que será más difícil de controlar por los responsables de la estrategia de suministro de fabricantes y propietarios de marca (Zhao et al., 2016).

Con estos antecedentes, presentamos un trabajo original y novedoso en el estudio del diseño de canales de distribución de artículos de gran consumo, y el impacto que sobre su estrategia tiene la presencia de un mercado paralelo de distribución de sus productos. Con ello, contribuimos a la literatura sobre el fenómeno por medio del análisis de tres aspectos relevantes.

Primero, la literatura ha localizado el origen del mercado paralelo principalmente en la diferencia de precios que se produce en aquellos productos de gran consumo que se comercializan en distintos mercados (Zhao et al., 2016), generando un flujo desde el mercado más económico hacia aquel de mayor precio (Cespedes et al., 1988). Como excepción, Antia et al. (2006) apunta como origen la escasez por incapacidad de satisfacer un exceso de demanda, y que genera compras en mercados donde está disponible.

Nuestro trabajo de investigación aporta al conocimiento del mercado paralelo un origen de gran importancia en el ámbito empresarial, aunque inédito en la literatura: el que se produce por un desabastecimiento estratégico, cuando el fabricante asigna determinados productos para cada intermediario en el diseño de la cobertura de sus clientes y utiliza distribuidores específicos y especializados para cada tipo (por ejemplo, B2B o B2C) (Coughlan et al., 2006). Esta especialización asigna un surtido de productos diferentes en función de la línea de distribución a la que pertenece cada intermediario y provoca la indisponibilidad de referencias concretas para determinados distribuidores, lo que puede originar negocios de mercado paralelo entre unas y otras líneas estratégicas.

Segundo, las soluciones que la literatura aporta al mercado paralelo las localizamos agrupadas en los siguientes tipos: 1) acciones encaminadas a reducir la brecha de precios por mercados para el mismo producto (Zhao et al., 2016), 2) desabastecimiento de aquellos distribuidores o canales por donde se localiza la fuga al mercado paralelo (Berman, 2004), ya que este tipo de acciones se inicia mediante un distribuidor oficial (Antia et al., 2004), y 3) gestionando la relación, donde tan solo localizamos un trabajo (Antia et al., 2006). Nuestra investigación ofrece una solución basada en enforcement que incide en la vía relacional y que aporta a la literatura una solución con un tratamiento casi inédito y con mucho potencial de desarrollo.

Porúltimo, dentro de la gestión de la relación que apuntamos como solución a la incidencia del mercado paralelo, el enforcement se muestra como un sistema disciplinario de medidas estructuradas y sintonizadas, destinadas a que los integrantes del canal se atengan a la estrategia trazada (Antia et al., 2006). El valor de esta contribución se mide en términos de desempeño para las organizaciones, ya que el cumplimiento de las normas contribuye a su éxito (Akerlof, 2016). Nuestro trabajo aporta a la literatura una peculiaridad no tratada: su efecto no lineal. Contrastaremos empíricamente si conforme aumentan los niveles en su aplicación se muestra más efectivo, situación también observada por Shaffer (1990) en el ámbito legislativo, donde encuentra que un aumento de las sanciones se traduce en un mayor cumplimiento de las normas, lo que aplicado al canal de distribución, ofrece una visión novedosa de su gobierno.

El aprovisionamiento mediante el mercado paralelo para un distribuidor oficial que no acepta la política de cobertura a clientes y surtido asignado y que no cumple sus acuerdos en el seno de la distribución oficial, lo sitúa en una relación de oportunismo impulsado por la estrategia del fabricante (Sa Vinhas y Heide, 2014). Este oportunismo es una variable central en la Teoría de Costes de Transacción (TCT) (Rindfleisch, 2019), y es el marco teórico en el que situamos nuestra investigación: en un entorno donde el aprovisionamiento mediante mercado paralelo está presente y es una opción para distribuidores oficiales que se enfrenten a la visión de la estrategia implantada ¿cómo debe gestionar el fabricante o propietario de la marca este oportunismo? Nos unimos a la literatura que analiza la gestión del oportunismo que supone la participación en el mercado paralelo en el marco de la TCT (Bergen et al., 1998; Dutta et al., 1994). 


\section{Revisión de la literatura e hipótesis}

Como parte del diseño del canal de distribución de productos de gran consumo es necesario adoptar una decisión estratégica: la elección de los mercados o segmentos considerados objetivo (Corey et al., 1989), en línea con el paradigma que señala que "uno de los principios fundamentales del marketing es la segmentación del mercado" (Coughlan et al., 2006; p. 17). Esta segmentación supone la elección de los intermediarios que compondrán el denominado canal de distribución oficial, situación que observamos especialmente en el caso de productos de gran consumo con marca de reconocido prestigio (Assmus y Wiese, 1995). Su implantación en el canal supone una restricción de tipo vertical.

Por tanto, el proceso de diseño del canal de distribución para artículos de gran consumo lleva aparejado el establecimiento de una distribución oficial asignada a un ámbito de actuación o territorio organizado de distribución (Corey et al., 1989), que podrá ser tanto un espacio físico o geográfico (Bergen et al., 1998), como un tipo de cliente (Dutta et al., 1999), o cualquier otro segmento coherente con el diseño del canal de distribución. Las políticas de cobertura de distribución seguidas por los fabricantes tienen el fin de proveer el producto mediante un intermediario especialista en cada tipo de cliente al que va destinado, proveyéndolo de servicios específicos para estos clientes (Coughlan et al., 2006). Esta especialización de los intermediarios tiene dos consecuencias: 1) la reducción de intermediarios competidores para un mismo producto (Dutta et al., 1999) y, 2) la dotación de un surtido específico en función del cliente o mercado al que va destinado, como parte del servicio especializado (Zhao et al., 2016).

La asignación de ámbitos o territorios de distribución es una estrategia del fabricante, pero, a su vez, es una protección para el distribuidor, que persigue tres objetivos básicos: 1) incentivar al distribuidor asignado mediante la maximización de la eficiencia de sus negocios (Dutta et al., 1994) recibiendo éste la totalidad del beneficio disponible en el territorio (Gundlach, 2013) a través de la restricción de entrada de otros distribuidores competidores (Heide et al., 1998); 2) especializar al distribuidor en su ámbito de actuación, lo que conduce a un mejor conocimiento y gestión del mercado asignado y, por extensión, a la mejora de la eficiencia de su función distribuidora para un determinado producto, lo que repercute igualmente en una mejora del servicio al cliente (Dutta et al., 1999) y, por último; 3) el establecimiento de pseudo-monopolios a través de la reducción de la competencia intramarca, incrementando con ello la rentabilidad del canal para la red oficial mediante la venta con beneficios monopolísticos en cada mercado asignado (Dutta et al., 1994).
En un escenario como el descrito surge una cuestión estratégica de primer orden: ¿qué opciones tiene o puede contemplar un distribuidor disconforme con la estrategia trazada por el fabricante que lo ha excluido de la accesibilidad a determinada gama de productos? La literatura señala como fuente de aprovisionamiento alternativa al canal oficial el mercado paralelo de distribución (Antia et al., 2004). En esta línea, las restricciones de acceso a la distribución pueden jugar un papel motivante a realizar transacciones de negocios en el mercado paralelo (Antia et al., 2006).

Un mercado paralelo es un canal alternativo al oficial en cuya comercialización intervienen agentes que están excluidos de la distribución oficial o, perteneciendo a ella, se desmarcan de ésta desarrollando actuaciones fuera de la misma (Cespedes et al., 1988). El mercado paralelo es alimentado por distribuidores oficiales que aumentan sus beneficios vendiendo a agentes externos a la línea de distribución oficial asignada (Antia et al., 2006). Así, se producen ventas de productos de marca por canales de distribución no autorizados a ello (Antia et al., 2004).

La restricción de surtido coarta el crecimiento o el poder atender mercados interesantes para el distribuidor excluido, de modo que la presencia de dicha restricción, se presenta como un estímulo para el desarrollo de prácticas de aprovisionamiento mediante el mercado paralelo (Antia et al., 2006). Por ello, planteamos la siguiente hipótesis.

$H_{1}$ : La participación activa del distribuidor oficial en el mercado paralelo está positivamente influida por las restricciones de surtido establecidas por la política de cobertura de clientes del fabricante.

Para que las organizaciones prosperen y tengan éxito, deben ser capaces de hacer cumplir las normas (Akerlof, 2016), por lo que "se requiere una mano visible o esfuerzos explícitos de gobierno para reducir la fricción inherente a las relaciones entre partes" (Kumar et al., 2011, p. 1). Así, tras haber diseñado las condiciones en las que se desarrollará la distribución oficial, estableciendo los segmentos de distribución y, en función de éstos, la política de cobertura de clientes (Coughlan et al., 2006), corresponde al fabricante establecer un sistema disciplinario para hacer cumplir con los aspectos descritos en la organización del canal (Antia et al., 2004). Este sistema comprenderá un conjunto de medidas predefinidas y estructuradas, destinadas a que los integrantes del canal de distribución se atengan a la estrategia trazada: el enforcement (Antia et al., 2006).

En el contexto de la organización del canal oficial de distribución, se define enforcement como "el conjunto de medidas con las que el fabricante asegura el cumplimiento del distribuidor en cuanto a las restricciones establecidas a 
su distribución, que incluyen penalizaciones económicas, litigios, ostracismo social o, en última instancia, ruptura y finalización de la relación oficial" (Antia et al., 2006; p. 93). Los autores describen tres dimensiones del concepto: 1) habilidad para detectar un incumplimiento; 2) velocidad con la que se reacciona ante este incumplimiento $\mathrm{y}, 3$ ) severidad con la que se aplica una acción correctiva ante dicho incumplimiento.

Primero, para tomar acciones correctivas el fabricante debe tener información de que se ha producido un incumplimiento ya que, en ocasiones, su detección es costosa e imperfecta (Dutta et al., 1999). Esta dimensión es conocida como habilidad para detectar el incumplimiento (Antia et al., 2006), donde se obtiene información y se desarrollan destrezas para percibir dicho incumplimiento mediante el diseño de sistemas específicos para detectarlo (Myers, 1999). Esta habilidad puede desarrollarse mediante la atenta observación de los integrantes de la distribución oficial, así como con la colaboración de agentes que se dediquen a vigilar el mercado (Antia et al., 2004). Por ejemplo, intermediarios oficiales con ventas anormalmente altas de producto, o disminución de compra singular del mismo puede significar que está vendiendo o comprando al margen de la red oficial. Para reforzar estas observaciones se pueden utilizar como vigilantes a aquellos distribuidores oficiales con los que se tiene una relación fluida o la propia fuerza de ventas del fabricante (Myers, 1999). En la actualidad también existen mecanismos de control y medios que permiten identificar la trazabilidad del producto, sabiendo en todo momento a quién se ha vendido y dónde se ha comprado, mediante el seguimiento de códigos EAN, números de serie, loteado del artículo o incluso las etiquetas RFID (Bandyopadhyay, 2010).

Segundo, otro aspecto definitorio del enforcement es la velocidad y certeza de su aplicación. Antia et al.(2006) señalan la velocidad como el tiempo que transcurre entre el momento en que el fabricante tiene conocimiento del incumplimiento y el momento en que aplica medidas. La certeza hace mención a la probabilidad de que el agente que ha incumplido recibirá su correspondiente reacción. Encontramos en la literatura que el enforcement es un concepto transversal estudiado por numerosas disciplinas, destacamos además del marketing, la psicología o la criminología. Sus múltiples tratamientos han conducido a la conclusión de que éste es más efectivo cuando combina la certeza de que va a ser aplicado con una mayor velocidad en su aplicación (Gray et al., 1982).

La tercera dimensión del enforcement es la severidad, que es la fuerza o intensidad del correctivo aplicado cuando se detecta un incumplimiento (Antia et al., 2006). Así, será tanto más efectivo en función de la severidad aplicada (Bergen et al., 1998). Antia y Frazier (2001) definen enforcement en términos de severidad, de tal manera que si no se percibe severidad en la aplicación de medidas disciplinarias éstas pierden eficacia en su capacidad disuasoria. La severidad es la dimensión que las empresas deben gestionar con mayor atención debido a que es su aspecto fundamental (Dutta et al., 1994).

Proponemos un efecto negativo del enforcement aplicado por el proveedor sobre la participación de sus distribuidores en el mercado paralelo. Asimismo, esperamos que dicho efecto sea no lineal. En la literatura sobre normas económicas, Akerlof (2016) plantea un juego de movimiento secuencial para dos jugadores, donde uno de ellos decide si acepta las normas y el segundo toma decisiones en torno a si sanciona los incumplimientos. Una solución eficaz sugerida consiste en elevar el nivel sancionador para evitar que el jugador que incumple sea reiterativo en su incumplimiento: mayores niveles de enforcement son más efectivos. También, en un entorno legislativo, Shaffer (1990) encuentra que el aumento marginal de las sanciones hace que el cumplimiento sea creciente.

Las teorías expuestas sugieren que sin enforcement los niveles de participación en el mercado paralelo serán los mayores, situación que se constata en entornos donde se toleran este tipo de acciones (Bergen et al., 1998). Pequeños incrementos en él apenas lo hacen relevante de modo que apenas si incidirá en la disminución del mercado paralelo, si bien, conforme aumente significativamente su nivel, su efecto será cada vez mayor. Primero, porque el enforcement se hace creíble para el distribuidor y, segundo, porque señala una preocupación del fabricante por invertir y controlar el mercado paralelo. En el ámbito de los impuestos (Mukhatar et al., 2001) y de las normativas de circulación (Henstridge et al., 1997), los efectos del enforcement sobre el cumplimiento de las normas también han presentado efectos no lineales marginalmente crecientes. Por ello proponemos las siguientes hipótesis:

$\mathrm{H}_{2}$ : La participación activa del distribuidor oficial en el mercado paralelo está negativamente influida por el enforcement del fabricante.

$H_{3}$ : El efecto negativo del enforcement sobre la participación activa del distribuidor oficial en el mercado paralelo aumenta para mayores niveles de aquel.

Por último, nos preguntamos si en presencia de políticas de restricción de surtido, con potencial para motivar a los intermediarios oficiales a reaccionar acudiendo al mercado paralelo, el enforcement modera dicha reacción. Aunque la literatura sugiere tratar el oportunismo detectado en el socio de negocios mediante socialización (Wathne y Heide, 2000), lo cierto es que, en canales de comercialización de productos de gran consumo, con presencia de marcas de gran poder, el vehículo más eficaz de influir externamente en el comportamiento del otro es el poder coercitivo (John, 1984), siendo más eficaz conforme más severa sea su aplicación (Antia y Frazier, 2001).

En este contexto, entendemos que si el enforcement cumple con las características necesarias para hacerlo 
efectivo, tanto en términos de habilidad de detección y velocidad de actuación (Antia et al., 2006), como en términos de severidad (Antia y Frazier, 2001), la tendencia a participar en el mercado paralelo como reacción a la restricción de surtido va a ser contenida por dicha disciplina. Gilliland et al. (2010) identifican el enforcement como el proceso mediante el cual el proveedor mantiene alineado al distribuidor en la estrategia, situación que planteamos como un efecto interacción entre la política de cobertura a clientes y enforcement, y que reflejamos en la siguiente hipótesis:

$\mathrm{H}_{4}: \quad \mathrm{El}$ efecto de las restricciones de surtido establecidas por la política de cobertura a clientes sobre la participación de los distribuidores oficiales en el mercado paralelo está negativamente influido por el enforcement del fabricante (moderación negativa).

\section{Análisis empírico}

\subsection{Escalas de medida}

La variable dependiente es la Participación Activa del Distribuidor en el Mercado Paralelo (PAC). Esta acción del distribuidor oficial es un ejercicio de oportunismo donde éste decide unilateralmente variar las condiciones acordadas del negocio que le ha sido asignado (Wathne y Heide, 2000). Para la obtención de escalas de medida se va a seguir el enfoque utilizado por Antia et al. (2006), mediante escalas adaptadas de oportunismo (Nunlee, 2005) (Tabla 1).
Tabla 1 Ítems de medición de las escalas PAC, RES y ENF

Los items han sido medidos utilizando una escala tipo Likert en un rango $1=$ muy en desacuerdo a $7=$ muy de acuerdo.
PAC (Participación activa del distribuidor oficial en el mercado paralelo):

La mayoría de las veces, abastecerse en el mercado paralelo es la mejor elección

Compra mercancía de esta marca en los mercados paralelos para beneficiar a sus clientes

Nunca es un error abastecerse del mercado paralelo

Saca producto al paralelo porque es una buena alternativa de rentabilidad para su empresa

Fuente: Antia et al., 2006; Dwyer y Oh, 1987; Nunlee, 2005

RES (Restricción de surtido inducida por el proveedor):

$>\quad$ El proveedor le niega la distribución de ciertos productos que "van" por otro canal

Fuente: Dutta et al., 1999

ENF_HAB (Habilidad de detección del fabricante ante un incumplimiento):

Este fabricante evalúa con relativa sencillez si se cumple con sus políticas de distribución

Este fabricante tiene buena información del volumen de ventas no autorizadas

Este fabricante puede decirse que es hábil en la detección de incumplimientos

ENF_VEL (Velocidad en la aplicación del enforcement)

Este fabricante ante un incumplimiento tiene una respuesta instantánea

Este fabricante toma acciones inmediatas cuando alguien no es diligente con lo acordado

Transcurre poco tiempo entre un incumplimiento y su respuesta

ENF SEV (Severidad en la aplicación del enforcement):

$>\quad$ Este fabricante tiene establecida una política destinada a que se cumplan los acuerdos con él.

Este fabricante es muy severo cuando se incumplen sus directrices.

Este fabricante es duro e intransigente respecto al desvío de producto al mercado no oficial 
La presencia de restricciones de surtido establecidas por la política de cobertura de clientes (RES) es medida a partir de un ítem que indaga sobre dicha condición en relación a si el distribuidor está excluido de la distribución de ciertos productos, que se asigna a otros distribuidores (Dutta et al., 1999) (Tabla 1). En este caso, y siguiendo los argumentos de Rossiter (2002) y Bergkvist y Rossiter (2007), se entiende que la restricción de surtido es un concepto claro, concreto y singular para dar respuesta mediante un solo ítem. Además, la información obtenida no habría sido más completa añadiendo más ítems al constructo.

Por su parte, el enforcement, cuya acción se expuso mediante tres dimensiones: 1) Habilidad desarrollada por el fabricante para detectar estos incumplimientos (ENF_HAB), 2) Velocidad en la aplicación desde que el incumplimiento es detectado (ENF_VEL) y 3) Severidad con la que es aplicado (ENF SEV), es medido a partir de las escalas de Antia et al. (2006) que se muestran en la Tabla 1.

\subsection{Obtención de datos}

La obtención de datos se ha realizado en el ámbito del sector del gran consumo, debido a que es un mercado caracterizado porque sus proveedores tienen implantada una estrategia multicanal para satisfacer la diversidad de su demanda, especialmente notorio en aquellos productos de reconocida marca en el mercado, donde observamos una política de cobertura a clientes mediante especialización de canales por tipo de cliente (restauración, intermediarios mayoristas o minoristas), que da lugar a la asignación por parte del proveedor de diferentes surtidos y servicios en función del distribuidor que atiende cada canal.

Las respuestas se obtuvieron a partir de una base de datos de distribuidores mayoristas españoles del mercado de gran consumo de alimentación y bebidas, a los que se les indicaba en el cuestionario que los destinatarios objeto de estudio debían cumplir la condición de ser intermediarios oficiales de un producto/marca y, por tanto, formar parte de la llamada red de distribución oficial, solicitando a aquellos distribuidores que no cumplieran con tal condición que no completasen el cuestionario. De igual forma, pedíamos respuesta instando a que pensaran en la marca de producto más prestigiosa o reconocida de entre aquellas que distribuían. Previamente, se realizó un pre-test, solicitando a ocho gerentes de distribución mayorista su respuesta mediante entrevista personal, lo que permitió afinar el cuestionario teniendo en cuenta el público objetivo.

La recogida de datos se hizo mediante el envío de correos electrónicos con un enlace web. En una primera fase, fueron enviados 3.193 correos electrónicos, donde se obtuvieron 103 respuestas válidas. En una segunda fase, se recurrió a la misma base de datos solicitando contestación a aquellos distribuidores que no lo habían hecho en la primera fase. Este segundo envío permitió agregar 78 respuestas, para un total de 181 respuestas válidas.

Para comprobar la presencia de posible sesgo de no respuesta, se analizó el momento en el que fueron facilitadas las contestaciones al cuestionario, dividiendo la muestra en dos submuestras. La comparación de ambas muestras se realizó mediante una prueba de muestras independientes (prueba $t$ de igualdad de medias). Los resultados muestran que no existen diferencias relevantes en el estudio en la comparación de las respuestas dadas en la primera fase con las obtenidas en la segunda, lo que sugiere inexistencia de sesgo de no respuesta (Armstrong y Overton, 1977).

\subsection{Fiabilidad y validez de las escalas de medida}

Para valorar la unidimensionalidad de los conceptos utilizados se realizó un análisis factorial confirmatorio (CFA) (Gerbing y Anderson, 1988) (Tabla 2). Se comprueba la fiabilidad compuesta de las escalas de medida mediante el indicador SCR, donde los valores superan en todos los casos el mínimo recomendado por Bagozzi y Yi (1988) de 0,60. Además, se observa validez convergente al ser la totalidad de cargas factoriales altamente significativas ( $\mathrm{t}$ mínimo es 6,38) (Bagozzi y Yi, 1988). 
Tabla 2 Resultados del análisis factorial confirmatorio (CFA)

Estadísticos del modelo de medición: $\chi_{(g l=59)}^{2}=115,77$; $G F I=0,90 ; S R M R=0,06$ $C F I=0,97 ; N F I=0,94$; IFI $=0,97$.

\begin{tabular}{|c|c|c|c|}
\hline Descripción del ítem & Estandarizado & Valor t & Fiabilidad \\
\hline $\begin{array}{l}\text { Participación activa distribuidor oficial en } \\
\text { mercado paralelo (PAC) }\end{array}$ & & & $\begin{array}{l}\mathrm{AVE}=0,46 \\
\mathrm{SCR}=0,77\end{array}$ \\
\hline PAC 1 & 0,64 & 8,29 & \\
\hline PAC 2 & 0,87 & 11,61 & \\
\hline PAC 3 & 0,51 & 6,38 & \\
\hline PAC 4 & 0,65 & 8,53 & \\
\hline $\begin{array}{l}\text { Habilidad detección incumplimientos (ENF } \\
\text { HAB) }\end{array}$ & & & $\begin{array}{l}\mathrm{AVE}=0,64 \\
\mathrm{SCR}=0,85\end{array}$ \\
\hline ENF_HAB 1 & 0,73 & 10,46 & \\
\hline ENF_HAB 2 & 0,76 & 11,12 & \\
\hline ENF_HAB 3 & 0,92 & 14,40 & \\
\hline Velocidad aplicación enforcement (ENF_VEL) & & & $\begin{array}{l}\mathrm{AVE}=0,78 \\
\mathrm{SCR}=0,91\end{array}$ \\
\hline ENF_VEL 1 & 0,90 & 14,86 & \\
\hline ENF_VEL 2 & 0,89 & 14,41 & \\
\hline ENF_VEL 3 & 0,85 & 13,51 & \\
\hline Severidad aplicación enforcement (ENF_SEV) & & & $\begin{array}{l}\mathrm{AVE}=0,65 \\
\mathrm{SCR}=0,84\end{array}$ \\
\hline ENF_SEV 1 & 0,73 & 10,40 & \\
\hline ENF_SEV 2 & 0,84 & 12,78 & \\
\hline ENF SEV 3 & 0,83 & 12,42 & \\
\hline
\end{tabular}

En la Tabla 3 se presentan los estadísticos descriptivos, las correlaciones, y los límites superiores o inferiores, según corresponda, de los intervalos de confianza. La variable enforcement (ENF) se construye como media de las tres dimensiones expuestas. Por otra parte, se confirma la validez discriminante de las escalas mediante los intervalos de confianza con \pm 2 errores estándar sobre la correlación entre constructos (phi), comprobando que el intervalo no incluye, en valor absoluto, el valor 1 (Anderson y Gerbing, 1988). 


\begin{tabular}{|c|c|c|c|c|c|c|}
\hline Tabla $\quad 3 \quad$ Estadísticos & Variable & Media & D. Estándar & 1 & 2 & 3 \\
\hline y límites de intervalos de & 1.- PAC & 2,26 & 1,32 & & $0,53^{(2)}$ & $-0,41^{(3)}$ \\
\hline confianza. & & & & & & \\
\hline (1) Correlaciones, (2) limite & 2.- RES & 3,19 & 2,07 & $0,36^{(1)}$ & & $0,28^{(2)}$ \\
\hline superior intervalo de & & & & & & \\
\hline confianza, (3) limite inferior & 3.- ENF & 4,03 & 1,43 & $-0,19^{(1)}$ & $0,07^{(1)}$ & \\
\hline
\end{tabular}

intervalo de confianza.

\subsection{Resultados}

Para corroborar las hipótesis se ha estimado una ecuación de regresión (1) utilizando mínimos cuadrados ordinarios:

(1) $P A C=b_{0}+b_{1} C 1 \_B E B+b_{2} C 2 \_P E R+b_{3} C 3 \_N O P$ $+b_{4} C 4 \_T M A+b_{5} C 5 \_A M B+b_{6} C 6 \_H O S+b_{7} C 7 \_M A Y$ $+b_{8} C 8 \_M I N+b 9 C 9 \_T D I+b 10 C 10 \_F A L+b 11 R E S+$ $b_{12} E N F+b_{13} E N F^{2}+b_{14} R E S x E N F+b_{15} R E S x E N F^{2}$

Para comprobar que el modelo teórico mantiene los resultados por encima de otros factores, se introducen en la regresión las siguientes variables de control: $\mathrm{C} 1 \_\mathrm{BEB}$ (categoría bebidas), C2_PER (alimentación perecedera), C3_NOP (alimentación no perecedera), C4_TMA (tamaño proveedor), C5_AMB (ámbito territorial de distribución), C6_HOS (cliente destino hostelería), C7_MAY (cliente destino mayoristas), C8_MIN (cliente destino minorista), C9_TDI (tamaño distribuidor) y C10_FAL (escasez global de producto).

Los conceptos del modelo principal son introducidos mediante índices construidos como valor medio de los ítems que los componen, siendo PAC la participación activa del distribuidor oficial en el mercado paralelo, RES la presencia de restricción de surtido inducida por el proveedor en la segmentación de la distribución y ENF el enforcement o medidas disciplinarias del proveedor para hacer cumplir su estrategia.

Para evitar la multicolinealidad esperada de la introducción de efectos interacción y cuadráticos, estos términos se construyen centrando previamente a la media las variables explicativas (RES y ENF) (Aiken y West, 1991). Comprobamos la ausencia de multicolinealidad mediante el examen del factor de inflación de la varianza obtenido en la regresión (FIV), donde se muestra que la totalidad de los valores quedan por debajo de la cifra crítica de 10 (Hair et al., 1999), siendo el más elevado de 4,78 (Tabla 4).

La Tabla 4 muestra los resultados obtenidos mediante el análisis de regresión jerárquica, donde el Modelo Teórico es confrontado con el mismo modelo sin efectos interacción, denominado Modelo de Efectos Principales y, por otro lado, con un modelo donde sólo se contemplan las variables de control definidas. La comparación del coeficiente de determinación lineal obtenido en los tres modelos permite concluir que el Modelo Teórico presenta un ajuste significativamente mejor que el resto. 
Tabla 4 Resultados de estimación del modelo.

La tabla muestra los resultados obtenidos mediante análisis de regresión jerárquica, donde el modelo principal es comparado con el mismo modelo sin efectos interacción o de efectos principales y con el modelo donde se incluyen las variables control definidas.

\begin{tabular}{|c|c|c|c|c|c|c|c|c|c|}
\hline \multirow[b]{2}{*}{ Variables } & \multirow[b]{2}{*}{ Coeficientes } & \multicolumn{2}{|c|}{ Variables Control } & \multicolumn{2}{|c|}{ Modelo Efectos Principales } & \multicolumn{3}{|c|}{ Modelo Teórico } & \multirow{2}{*}{$\begin{array}{c}\text { Colinealidad } \\
\text { FIV }\end{array}$} \\
\hline & & Estand. & Signif. & Estand. & Signif. & Estand. & Signif. & No Estand. & \\
\hline Constante & b0 & & & & & & & 1,22 & \\
\hline \multicolumn{10}{|l|}{ Control: } \\
\hline C1_BEB & $b_{1}$ & 0,21 & 0,26 & 0,23 & 0,17 & 0,18 & 0,28 & 0,45 & 4,78 \\
\hline C2_PER & $\mathrm{b}_{2}$ & 0,09 & 0,58 & 0,14 & 0,30 & 0,11 & 0,39 & 0,34 & 3,19 \\
\hline C3_NOP & $b_{3}$ & 0,03 & 0,83 & 0,15 & 0,28 & 0,10 & 0,49 & 0,28 & 3,34 \\
\hline C4_TMA & $\mathrm{b}_{4}$ & $-0,02$ & 0,85 & $-0,05$ & 0,53 & $-0,01$ & 0,93 & $-0,01$ & 1,13 \\
\hline C5_AMB & $b_{5}$ & $-0,03$ & 0,79 & 0,08 & 0,41 & 0,08 & 0,42 & 0,12 & 1,70 \\
\hline C6_HOS & $\mathrm{b}_{6}$ & $-0,02$ & 0,82 & $-0,01$ & 0,90 & $-0,02$ & 0,82 & $-0,06$ & 1,34 \\
\hline C7_MAY & $\mathrm{b}_{7}$ & 0,02 & 0,84 & $-0,01$ & 0,95 & $-0,00$ & 0,99 & $-0,00$ & 1,36 \\
\hline C8_MIN & $\mathrm{b}_{8}$ & 0,16 & 0,11 & 0,13 & 0,17 & 0,12 & 0,19 & 0,30 & 1,43 \\
\hline C9_TDI & $\mathrm{b}_{9}$ & 0,10 & 0,31 & 0,10 & 0,29 & 0,19 & 0,06 & 0,60 & 1,78 \\
\hline C10_FAL & $\mathrm{b}_{10}$ & 0,13 & 0,18 & $-0,05$ & 0,55 & 0,01 & 0,92 & 0,01 & 1,49 \\
\hline \multicolumn{10}{|l|}{ Conceptuales: } \\
\hline RES & $b_{11}$ & & & 0,46 & 0,00 & 0,54 & 0,00 & 0,34 & 2,07 \\
\hline ENF & $\mathrm{b}_{12}$ & & & $-0,23$ & 0,01 & $-0,35$ & 0,00 & $-0,30$ & 1,54 \\
\hline $\mathrm{ENF}^{2}$ & $\mathrm{~b}_{13}$ & & & & & $-0,29$ & 0,00 & $-0,15$ & 1,43 \\
\hline RES x ENF & $\mathrm{b}_{14}$ & & & & & $-0,18$ & 0,06 & $-0,07$ & 1,55 \\
\hline RES $x \mathrm{ENF}^{2}$ & $\mathrm{~b}_{15}$ & & & & & $-0,22$ & 0,07 & $-0,04$ & 2,64 \\
\hline \multicolumn{10}{|l|}{ Estadísticos: } \\
\hline $\begin{array}{c}\mathrm{R}^{2}\left(\mathrm{R}^{2}\right. \\
\text { ajustada) }\end{array}$ & & & & & & & $0,34(0,25)$ & & \\
\hline $\begin{array}{l}\text { Valor F } \\
\text { (Pro.F) }\end{array}$ & & & & & & & $22,55(0,00)$ & & \\
\hline $\begin{array}{l}\text { Camb. } \mathrm{R}^{2} \\
\text { ( } \mathrm{R}^{2} \text { ajus.) }\end{array}$ & & & & & & & $0,06(0,05)$ & & \\
\hline $\begin{array}{c}\text { F camb. R2 } \\
\text { (Pro.F) }\end{array}$ & & & & & & & $3,79(0,01)$ & & \\
\hline
\end{tabular}


Para analizar el efecto de las variables independientes sobre la participación activa del distribuidor en el mercado paralelo, y puesto que el modelo incluye efectos no lineales e interacción, se sigue el procedimiento propuesto por Aiken y West (1991) y Jaccard et al. (1990). Así, el efecto de RES sobre PAC, en presencia de ENF, se describe con la siguiente función (2):

$\frac{\partial P A C}{\partial R E S}=b_{11}+b_{14} E N F+b_{15} E N F^{2}$

En el estudio de la extensión total del rango de una variable se consideran valores bajos a aquellos con un valor de -1 desviación típica y, como valores altos, +1 desviación típica (Aiken y West, 1991). En la Figura 1 se representa el efecto que RES tiene sobre PAC para distintos valores de
ENF. Apreciamos que la curva que representa la presencia de una política de restricción de surtido (RES) tiene pendiente significativamente positiva, tanto para valores bajos como altos de enforcement (ENF), lo que nos conduce a corroborar la hipótesis $\mathrm{H}_{1}$. La participación en el mercado paralelo aumenta para mayores niveles de presencia de una política de restricción de surtido. Observamos asimismo que el efecto de RES sobre PAC es menor cuando ENF está fijado a valores altos $(\beta=0,15 ; \mathrm{t}=2,74 ; \mathrm{p}=0,01)$, frente a cuando los valores de ENF son bajos, obteniéndose en este caso un mayor efecto de RES sobre PAC $(\beta=0,36 ; \mathrm{t}=6,60$; $\mathrm{p}=0,00)$. La diferencia de dichos efectos es significativa $(\beta=-0,21 ; \mathrm{t}=-2,34 ; \mathrm{p}=0,02)$, por lo que podemos concluir que el enforcement modera el efecto de RES sobre PAC. Este resultado nos conduce a corroborar la hipótesis $\mathrm{H}_{4}$.

Figura 1 Efectos de RES sobre PAC para niveles bajos y altos de ENF.

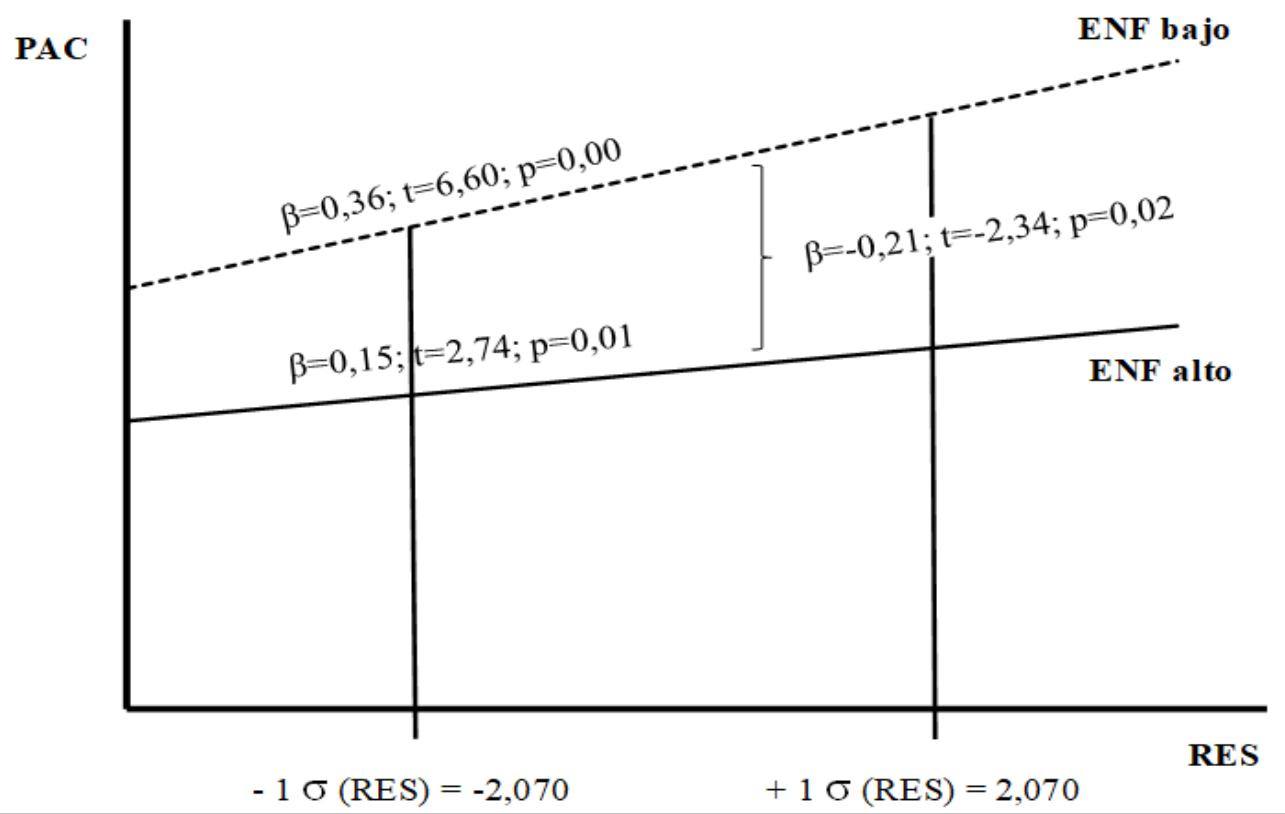

De manera análoga, aplicamos la misma técnica para analizar el efecto marginal que ENF tiene sobre PAC, cuando la política de restricción de surtido (RES) se mueve en valores medios ( $\mathrm{RES}=0$, al estar las variables centradas a la media). Situación que describe la siguiente expresión (3):

$\frac{\partial P A C}{\partial E N F}=b_{12}+2 b_{13} E N F$

La Figura 2 presenta los resultados obtenidos, donde apreciamos que cuando ENF se mueve en valores bajos su efecto es no significativo $(\beta=0,11 ; \mathrm{t}=0,88 ; \mathrm{p}=0,38)$. Por otra parte, cuando ENF alcanza altos valores sí encontramos significatividad $(\beta=-0,72 ; \mathrm{t}=-4,30 ; \mathrm{p}=0,00)$, además de cambiar el sentido de la pendiente, lo que nos indica que el impacto del mencionado enforcement (ENF) sobre la participación activa en el mercado paralelo (PAC) sólo es efectivo para valores altos. Además, el cambio de valor en enforcement de niveles bajos a altos también muestra significatividad $(\beta=-0,83 ; \mathrm{t}=-3,26 ; \mathrm{p}=0,00)$, corroborando las hipótesis $\mathrm{H}_{2} \mathrm{y} \mathrm{H}_{3}$. 
Figura 2 Efectos de ENF sobre PAC para niveles medios de RES.

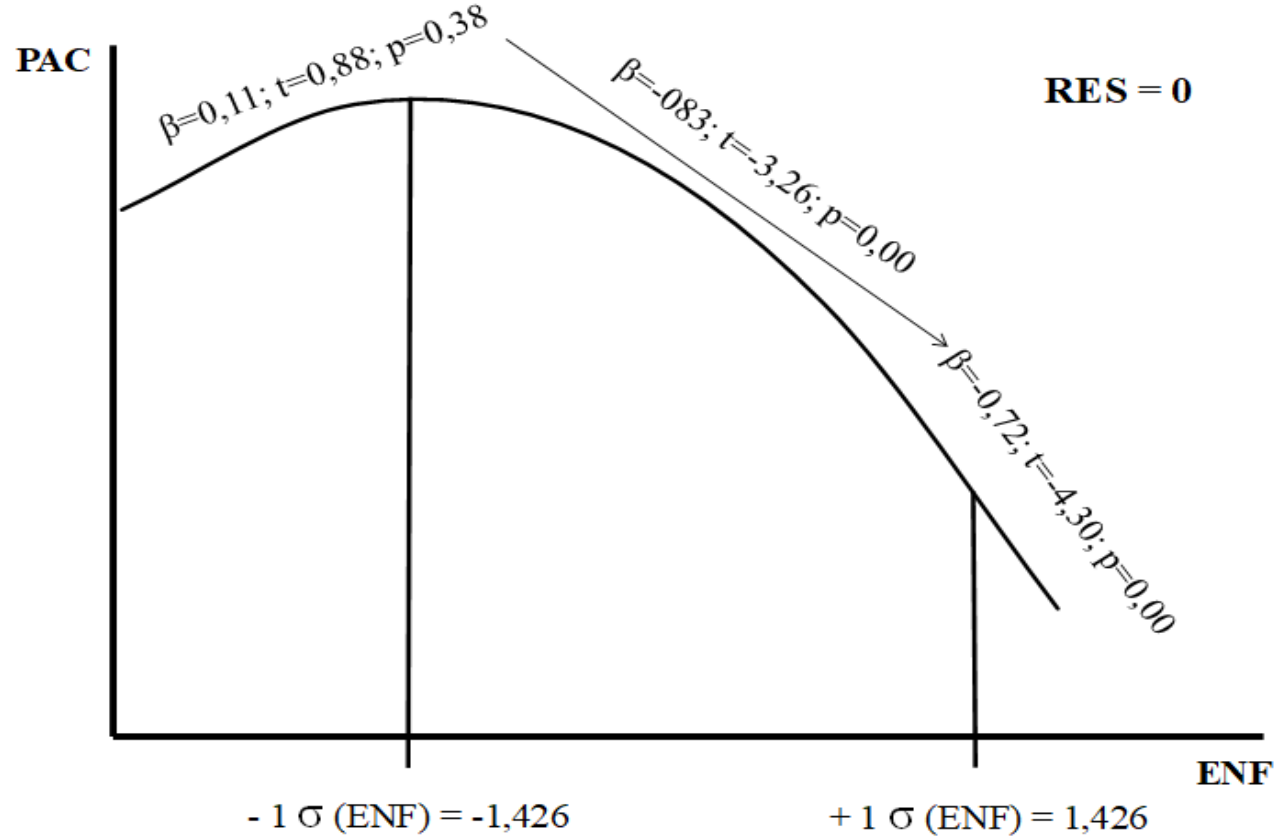

\section{Conclusiones y recomendaciones a la gestión empresarial}

El estudio del efecto que la política de restricción de surtido tiene sobre la participación en el mercado paralelo, así como las medidas disciplinarias destinadas a hacer cumplir con la organización de la red oficial, aporta nuevas perspectivas de análisis en el estudio de los canales de distribución de gran consumo.

Se constata que cuando la política de cobertura por clientes que se aplica a un distribuidor oficial consiste en calificarlo para distribuir unos productos determinados de la cartera del proveedor, excluyéndolo de la distribución de otros, se puede esperar en ocasiones una reacción de disconformidad con la organización del canal. Dicho desacuerdo es susceptible de dirigir al distribuidor oficial al mercado paralelo para proveerse y así poder asistir a aquellos clientes que la estrategia de distribución del canal le ha negado. La intención es la de aumentar unilateralmente la cuota de beneficios asignada, que es un comportamiento abordado por la literatura sobre oportunismo (ver Wathne y Heide, 2000) y cuya solución ha sido mayoritariamente tratada desde la perspectiva de las medidas disciplinarias.

Así, el proveedor ha de defender la organización del canal ideado en el seno de su estrategia de distribución mediante el despliegue de una serie de medidas disciplinarias o enforcement destinadas a hacer que este diseño estratégico se cumpla. El análisis empírico muestra como este conjunto de medidas es eficaz a la hora de reducir la propensión a participar en el mercado paralelo de los miembros de la red oficial y, por tanto, reduce la tendencia oportunista.
En última instancia, los resultados dan respuesta a la última cuestión de investigación planteada: ¿Es eficaz el enforcement del proveedor para disuadir de la participación en el mercado paralelo como consecuencia de la política de cobertura por clientes basada en la restricción de surtido? La respuesta a este interrogante es afirmativa, el enforcement es efectivo.

Por tanto, las recomendaciones para la gestión empresarial son las de acompañar la estrategia de diseño del canal con un conjunto de medidas disciplinarias destinadas a mantener el orden en la distribución oficial.

Estas medidas disciplinarias, como hemos visto, han de reunir determinadas características que hemos definido como dimensiones necesarias para la eficacia del enforcement. En primer lugar, el proveedor ha de desarrollar habilidades para detectar los incumplimientos de su red oficial, y no es tarea fácil. Los incumplimientos de las normas suelen ir acompañados de ocultación por parte de los incumplidores, correspondiendo al líder de la distribución oficial, el proveedor, desarrollar sistemas que le faciliten la información necesaria para tomar medidas.

La velocidad o prontitud con la que se reacciona y se toman medidas ante un incumplimiento de la estrategia oficial es otro de los requisitos necesarios para que un enforcement se muestre eficaz. En este sentido, es recomendable tener prediseñado un conjunto de medidas para reducir tiempos en su aplicación, y así conseguir que cada contingencia tenga una rápida y adecuada respuesta. 
El último aspecto que ha de cumplir el conjunto de medidas disciplinarias es el de ser lo suficientemente severo. En este sentido, algunos autores consideran el enforcement en función de la severidad que aplican (Antia y Frazier, 2001), por lo que entendemos que la falta de severidad es equiparable a la omisión de tomar medidas.

Destacamos en nuestro trabajo dos importantes contribuciones teóricas. En primer lugar, introducimos como motivante del mercado paralelo la política de cobertura por clientes mediante la restricción de surtido que un proveedor hace en el diseño de su estrategia de distribución mediante un canal oficial. Esta situación no ha sido tratada con anterioridad en la literatura como origen de la participación en el mercado paralelo, añadiendo una nueva dimensión al oportunismo exhibido por los agentes que participan en el mismo.

Por otra parte, destacamos como contribución la presentación y difusión de un análisis empírico que se une a aquellos que han refrendado la necesidad de un sistema estructurado y ordenado de medidas disciplinarias. La literatura nos ha mostrado el enforcement como una reacción eficaz en situaciones con potencial para comportamientos oportunistas (John, 1984).

\section{Limitaciones y futuras investigaciones}

Una de las limitaciones que localizamos en nuestro estudio es la óptica desde la que se analiza el impacto de la restricción de surtido y la disciplina en el canal de distribución, que podría ser más completa obteniendo datos de diada y analizando simultáneamente el impacto en la relación existente entre el intermediario oficial y el proveedor responsable del diseño del canal.

Aunque otros estudios que tratan la misma problemática presentan tamaños muestrales menores o muy parecidos al nuestro (Antia et al., 2006; Bergen et al., 1998), entendemos que un análisis empírico con un mayor tamaño muestral al utilizado contribuiría a reforzar en gran medida los hallazgos expuestos en nuestra investigación.

El progresivo avance e implantación de las nuevas tecnologías ha supuesto un escenario diferente para el mundo de los negocios del que no han quedado exentos los canales de distribución. Las TIC también afectan al diseño del canal y todos sus aspectos asociados, ya que la accesibilidad a la información deja de ser una barrera o un coste. El impacto que tienen estas nuevas tecnologías en las restricciones impuestas al intermediario o el impacto del enforcement no ha tenido un tratamiento específico en nuestro trabajo, situación que invitamos a corregir.
Para concluir, las últimas décadas de estudio han situado las relaciones entre los agentes como un elemento no sólo relevante sino fundamental dentro del diseño y gestión del canal de distribución. Nuestro estudio se ha enmarcado dentro de los canales de distribución de productos de gran consumo y ha obviado el aspecto social del canal como decisión consecuente con lo observado en la literatura (Frazier, 1999). Sin embargo, consideramos de interés entender cómo funcionan las relaciones en este ámbito y, sobre todo, cómo se gestionan sus distintos aspectos en este tipo de canales de distribución. Así, entendemos que un estudio de la relación conduciría, o bien a confirmar lo apuntado por la literatura, o bien a abrir una nueva línea de investigación en este ámbito.

\section{Referencias bibliográficas}

1. Aiken, L.S. and West, S.G. (1991). Multiple regression: Testing and interpreting interactions. Thousand Oaks, California: Sage Publications, Inc.

2. Akerlof, R. (2016). Anger and enforcement. Journal of Economic Behavior \& Organization, 126, 110-124.

3. Anderson, J.C. and Gerbing, D.W. (1988). Structural equation modeling in practice: $\mathrm{A}$ review and recommended two-step approach. Psychological Bulletin, 103 (3), 411-423.

4. Antia, K.D., Bergen, M.E. and Dutta, S. (2004). Competing with gray markets. MIT Sloan Management Review, 46 (1), 62-71.

5. Antia, K.D., Bergen, M.E., Dutta, S. and Fisher, R.J. (2006). How does enforcement deter gray market incidence? Journal of Marketing, 70 (January), 92-106.

6. Antia, K.D. and Frazier, G.L. (2001). The severity of contract enforcement in interfirm channel relationships. Journal of Marketing, 65 (October), 67-81.

7. Armstron, J.S. and Overton, T.S. (1977). Estimating nonresponse bias in mail Surveys. Journal of Marketing Research, 14 (3), 396-402.

8. Assmus, G. and Wiese, C. (1995). How to address the gray market threat using price coordination. Sloan Management Review, 36 (3, Spring), 31-41.

9. Bagozzi, R.P. and Yi, Y. (1988). On the evaluation of structural equation models. Journal of the Academy of Marketing Science, 16 (1), 74-94.

10. Bandyopadhyay, S. (2010). The internet and gray marketing. The International Business \& Economics Research Journal, 9 (6), 95-101. 
11. Bergen, M.E., Heide, J.B. and Dutta, S. (1998). Managing gray markets through tolerance of violations: A transaction cost perspective. Managerial and Decision Economics, 19 (3), 157-165.

12. Bergkvist, L. and Rossiter, J.R. (2007). The predictive validity of multiple-item versus single-item measures of the same constructs. Journal of Marketing Research, 44 (2), 175-184.

13. Berman, B. (2004). Strategies to combat the sale of gray market goods. Business Horizons, 47 (4), 51-60.

14. Cao, Q. and Zhang J. (2019). Gray market's product quality in the circular economy era. International Journal of Production Research, Vol. 58 (1), 338-331.

15. Cespedes, F.V., Corey, E.R. and Rangan, V.K. (1988). Gray markets: Causes and cures. Harvard Business Review, July-August, 75-82.

16. Corey, E.R., Cespedes, F.V. and Rangan, V.K. (1989). Going to market: Distribution systems for industrial products. Harvard Business School, First edition.

17. Coughlan, A.T., Anderson, E., Stern, L.W. and ElAnsary, A.I. (2006). Marketing channels. Prentice Hall, Seventh edition.

18. Dutta, S., Bergen, M. and John, G. (1994). The governance of exclusive territories when dealers can bootleg. Marketing Science, 13 (1), 83-99.

19. Dutta, S., Heide, J.B. and Bergen, M. (1999). Vertical territory restrictions and public policy: Theories and industry evidence. Journal of Marketing, 63 (October), 121-134.

20. Frazier, G.L. (1999). Organizing and managing channels of distribution. Journal of the Academy of Marketing Science, 27 (2), 226-240.

21. Gerbing, D.W. and Anderson, J.C. (1988). An updated paradigm for scale development incorporating unidimensionality and its assessment. Journal of Marketing Research, 25 (2), 186-192.

22. Gilliland, D.I., Bello, D.C. and Gundlach, G.T. (2010). Control-based channel governance and relative dependence. Journal of the Academy of Marketing Science, 38 (4), 441-455.
23. Gray, L.N., Alfred C. M., David A.W. and Menke, B. (1982). A game theoretical analysis of the components of punishments. Social Psychology Quarterly, 45 (4), 206-212.

24. Gudigantala, N. and Bicen, P. (2019). Do consumers' ethical judgments matter for purchase intentions in online gray markets?. Journal of Marketing Development and Competitivenes, Vol. 13 (4), 27-43.

25. Gundlach, G.T. (2013). Evolving changes in consumer shopping, marketing distribution and competitive interaction. The Antitrust Bulletin, 58 (1), 1-18.

26. Hair, J.F. Jr., Anderson, R.E., Tatham, R.L. and Black, W.C. (1999). Análisis multivariante. Prentice Hall, 5 edición.

27. Heide, J.B. (1994). Interorganizational governance in marketing channels. Journal of Marketing, 58 (January), 71-85.

28. Heide, J.B., Dutta, S. and Bergen, M. (1998). Exclusive dealing and business efficiency: Evidence from industry practice. Journal of Law and Economics, 41 (2), 387408.

29. Henstridge, J., Homel, R. and Mackay, P.M. (1997). The long-term of random breath testing in four Australian States: A time series analysis. Australian Government: Australian Transport Safety Bureau.

30. Jaccard, J.J., Turrisi, R. and Wan, C.K. (1990). Interaction effects in multiple regression. Sage Publications, Newbury Park, CA.

31. John, G. (1984). An empirical investigation of some antecedents of opportunism in a marketing channel. Journal of Marketing Research, 21 (3), 278-289.

32. Kumar, A., Heide, J.B., and Wathne, K.H. (2011). Performance implications of mismatched governance regimes across external and internal relationships, Journal of Marketing, 75 (March), 1-17.

33. Mukhtar, M.A., Cecil, H.W. and Knoblett, J.A. (2001). The effects of tax rates and enforcement policies on taxpayer compliance: A study of self-employed taxpayers. Atlantic Economic Journal, 29 (2), 186-202.

34. Myers, M.B. (1999). Incidents of gray market activity among U.S. exporters: Occurrences, characteristics, and consequences. Journal of International Business Studies, 30 (1), 105-126. 
35. Nunnlee, M.P. (2005). The control of intra-channel opportunism through the use of inter-channel communication. Industrial Marketing Management, 34, 515-525.

36. Rindfleisch, A. (2019). Transaction cost theory: Past, present and future. Academy of Marketing Science Review, 1-13.

37. Rossiter, J.R. (2002). The C-OAR-SE procedure for scale development in marketing. International Journal of Research in Marketing, 19, 305-335.

38. Sa Vinhas, A. and Heide, J.B. (2014). Forms of competition and outcomes in dual distribution channels: The distributor's perspective. Marketing Science, 34 (1), 1-16
39. Shaffer, S. (1990). Regulatory compliance with nonlinear penalties, Journal of Regulatory Economics, 2 (1), 99-103.

40. Wathne, K.H. and Heide, J.B. (2000). Opportunism in interfirm relationships: Forms, outcomes, and solutions. Journal of Marketing, 64 (October), 36-51.

41. Zhao, K., Zhao, X. and Deng, J. (2016). An empirical investigation of online gray markets. Journal of Retailing, 92 (4), 397-410. 
ANEXO: Resumen de datos obtenidos.

Tabla: resumen puntuaciones obtenidas por ítem (181 datos)

Los items han sido medidos utilizando una escala tipo Likert en un rango $1=$ muy en desacuerdo a $7=$ muy de acuerdo

\begin{tabular}{|c|c|c|c|c|c|c|c|c|}
\hline Descripción del ítem & 1 & 2 & 3 & 4 & 5 & 6 & 7 & NS/NC \\
\hline \multicolumn{9}{|c|}{ Participación activa distribuidor oficial en mercado paralelo (PAC) } \\
\hline PAC 1 & 79 & 26 & 19 & 21 & 15 & 14 & 7 & 0 \\
\hline PAC 2 & 121 & 24 & 8 & 14 & 4 & 5 & 5 & 0 \\
\hline PAC 3 & 78 & 22 & 12 & 32 & 6 & 13 & 15 & 3 \\
\hline PAC 4 & 103 & 32 & 8 & 14 & 6 & 9 & 1 & 8 \\
\hline \multicolumn{9}{|c|}{ Restricción de surtido inducida por el proveedor (RES) } \\
\hline RES 1 & 55 & 30 & 15 & 21 & 17 & 23 & 17 & 3 \\
\hline \multicolumn{9}{|c|}{ Habilidad detección incumplimientos (ENF_HAB) } \\
\hline ENF_HAB 1 & 9 & 18 & 19 & 33 & 37 & 37 & 16 & 12 \\
\hline ENF_HAB 2 & 23 & 32 & 23 & 35 & 16 & 16 & 22 & 14 \\
\hline ENF_HAB 3 & 13 & 22 & 25 & 36 & 30 & 19 & 21 & 15 \\
\hline \multicolumn{9}{|c|}{ Velocidad aplicación enforcement (ENF_VEL) } \\
\hline ENF_VEL 1 & 22 & 25 & 17 & 29 & 31 & 23 & 19 & 15 \\
\hline ENF_VEL 2 & 17 & 21 & 22 & 35 & 34 & 23 & 15 & 14 \\
\hline ENF_VEL 3 & 17 & 20 & 22 & 37 & 34 & 19 & 15 & 17 \\
\hline \multicolumn{9}{|c|}{ Severidad aplicación enforcement (ENF_SEV) } \\
\hline ENF_SEV 1 & 20 & 25 & 17 & 20 & 18 & 38 & 28 & 15 \\
\hline ENF_SEV 2 & 20 & 26 & 23 & 32 & 24 & 24 & 19 & 13 \\
\hline ENF_SEV 3 & 31 & 21 & 15 & 25 & 18 & 28 & 29 & 14 \\
\hline
\end{tabular}

